\title{
Hypoxia during embryonic development increases energy metabolism in normoxic juvenile chicks
}

\author{
Lara do Amaral-Silva a,b,1, Carolina da S. Scarpellini a,b,1, Paula Andrea Toro-Velasquez a , \\ Marcia H.M.R. Fernandes ${ }^{c}$, Luciane H. Gargaglioni ${ }^{\mathrm{a}, \mathrm{b}}$, Kênia C. Bícego ${ }^{\mathrm{a}, \mathrm{b}, *}$ \\ a Department of Animal Morphology and Physiology, College of Agricultural and Veterinarian Sciences, São Paulo State University, Jaboticabal, São Paulo 14884-900, Brazil \\ b National Institute of Science and Technology - Comparative Physiology (INCT-Fisiologia Comparada), Brazil \\ c Department of Animal Science, College of Agricultural and Veterinarian Sciences, São Paulo State University, Jaboticabal, São Paulo 14884-900, Brazil
}

\section{A R T I C L E I N F O}

\section{Article history:}

Received 8 October 2016

Received in revised form 1 March 2017

Accepted 5 March 2017

Available online 7 March 2017

\section{Keywords:}

Body temperature

Hypoxic incubation

Oxygen consumption

Phenotypic plasticity

Thermal preference

\begin{abstract}
A B S T R A C T
Environmental changes during perinatal development can affect the postnatal life. In this sense, chicken embryos that experience low levels of $\mathrm{O}_{2}$ over a specific phase of incubation can have their tissue growth reduced and the ventilatory response to hypoxia blunted, at least until hatching. Additionally, exposure to low level of $\mathrm{O}_{2}$ after birth reduces the thermogenesis as well. In the present study, we tested the hypothesis that hypoxia over the third week of incubation affects the thermoregulation of juvenile chicks at an age when thermogenesis is already expected to be well-developed. To this end, we measured body temperature ( $\mathrm{Tb})$ and oxygen consumption $\left(\dot{\mathrm{V}} \mathrm{O}_{2}\right)$ under acute hypoxia or different ambient temperatures (Ta) of 1 and 10 day-old chicks that have been exposed to $21 \% \mathrm{O} 2$ for entire incubation ( $\mathrm{Nx}$ ) or to $15 \% \mathrm{O}_{2}$ in the last week of incubation ( $\mathrm{Hx}$ ). We also assessed the thermal preference under normoxia or acute hypoxia of the older chicks from both incubation groups in a thermocline. Hypoxia over incubation reduced growth but did not affect the cold-induced thermogenesis in hatchlings. Regarding the juvenile $\mathrm{Hx}$, present data indicate a catch up growth with higher resting $\dot{V} \mathrm{O}_{2}$, a thermal preference for warmer Tas and a possible higher thermal conductance. In conclusion, our results show that hypoxia over the third week of incubation can affect the thermoregulation at least until 10 days after hatch in chickens.
\end{abstract}

(c) 2017 Elsevier Inc. All rights reserved.

\section{Introduction}

Hypoxia induces decrease in metabolic rate $\left(\mathrm{V}_{2}\right)$ and body temperature $(\mathrm{Tb})$ and increase in ventilation, which seems to minimize the imbalance between oxygen supply and demand. These responses are observed in newborns and adults of several species (Gautier, 1996; Bicego et al., 2007; Mortola, 2009; Mortola and Maskrey, 2011) whose metabolic suppression is accompanied by increase in autonomic (Tattersall and Milsom, 2003) and behavioral (Mortola and Feher, 1998; Bicego et al., 2007; Mortola and Maskrey, 2011) heat loss responses.

During prenatal stages, the hypoxic metabolic drop is observed mainly as depression of tissue growth because at this phase growth is

Abbreviations: BM, body mass; H0, hatchlings (between 12 and 20 hs); H10, 10 dayold chicks; Hx, hypoxic incubation (last week, 15\% O2); Nx, normoxic incubation (21\% O2); Ta, ambient temperature; Tb, body temperature; $\dot{V} 0_{2}$, metabolic rate assessed as the rate of oxygen consumed by the animal.

* Corresponding author at: Via de acesso Paulo Donato Castellane s/n, 14884-900, Departamento de Morfologia e Fisiologia Animal, Faculdade de Ciências Agrárias e Veterinárias, Universidade Estadual Paulista Júlio de Mesquita Filho, Jaboticabal, SP, Brazil.

E-mail addresses: keniacb@yahoo.com.br, keniacb@fcav.unesp.br (K.C. Bícego).

1 These authors contributed equally to this work. the most energy-demanding function, which can lead to immaturity of organs/systems in hatchlings (Mortola and Cooney, 2008; Mortola, 2009; Mortola and Awam, 2010). The drop in growth is also observed during non-hypoxic reduction of metabolic rate such as cold exposure over incubation (Mortola and Toro-Velasquez, 2013).

In contrast, after birth thermogenesis becomes the principal source of energy expenditure and its inhibition is the main factor involved in hypoxia-induced metabolic depression (Mortola and Maskrey, 2011). The opposite response (increase in energy expenditure), however, is observed during cold exposure to avoid hypothermia (cf. Bicego et al., 2007). These facts indicate that, although these two stressors (hypoxia and cold) have similar effects on metabolic rate during prenatal stages, in post-natal life their effects are opposite because of the establishment of endothermy (Szdzuy et al., 2008). In this context, it is interesting to note that hypoxia, but not cold exposure, during incubation induces reduced ventilatory response to low oxygen in chicken hatchlings (Mortola and Toro-Velasquez, 2013), indicating a specificity of the hypoxic stimulus to the chemosensory development.

All the studies mentioned above addressed morphophysiogical responses during pre-hatching phases or in the first day after hatching. It is possible that exposure to low levels of $\mathrm{O} 2$ over embryogenesis can cause not only short, but also prolonged morphophysiological 
alterations. The interest in postnatal consequences caused by environmental changes throughout perinatal development is because such alterations during critical phases can induce responses of genes that lead to different phenotypes later in life (Okubo and Mortola, 1989; Snyder et al., 1984). In the case of hypoxia, the phase of embryonic development that the animal is exposed to, is crucial to determine possible phenotypic changes (Chan and Burggren, 2005).

Because in birds the last week of incubation is a critical phase for some organs/system and thermogenesis maturation (Chan and Burggren, 2005; Ferner and Mortola, 2009; Szdzuy et al., 2008), we hypothesize that low levels of $\mathrm{O} 2$ over the third week of incubation would affect thermoregulation of chicks at the second week of life, i.e., when they are already expected to have a well-developed thermogenesis (Tzschentke and Nichelmann, 1999). To test this hypothesis, we measured $\mathrm{Tb}$ and oxygen consumption (index of thermogenesis) under acute hypoxia or different ambient temperatures (Ta) of 1 and 10 dayold chicks that have been exposed to $15 \% \mathrm{O} 2$ in the last week of incubation. We also measured the thermal preference in normoxia or acute hypoxia of the older chicks from both incubation groups.

\section{Methods}

\subsection{Animals}

Freshly laid fertilized eggs of lineage Cobb were obtained from a local supplier (Globoaves, Itirapina, SP, Brazil). All the eggs were weighed at day 0 , incubated at temperature of $37.5{ }^{\circ} \mathrm{C}$ and $60 \%$ of relative humidity and rotated every $2 \mathrm{~h}$. All of those parameters were controlled by sensors inside the incubators. One group of eggs remained under normoxia for the entire incubation $(21 \% \mathrm{O} 2$; $\mathrm{Nx})$, whereas another group was transferred into a hypoxic incubator (15\% $\mathrm{O} 2$; $\mathrm{Hx}$ ) between day 12 and day 18 of incubation. The desired level of hypoxia $(15 \% 02)$ was obtained by pushing into the incubator a small stream of N2 (0.2-0.4 mL/min; White Martins, Osasco, SP, Brazil) controlled by a flowmeter and the $\mathrm{O} 2$ concentration was monitored continuously by an $\mathrm{O} 2$ analyzer (Sensepoint XCD, Honeywell, USA). The incubator was equipped with three thermometers disposed in strategic points and one of them was very close to the nearest egg to the N2 leaking to make sure that the introduction of compressed gas would not alter the temperature of the incubator. On the 19th day all eggs were transferred to a normoxic hatcher $\left(37.5^{\circ} \mathrm{C}\right)$. After hatchlings were dry, they were transferred to chambers (Premium Ecológica, Belo Horizonte, Brazil) with controlled temperature $\left(33-31^{\circ} \mathrm{C}\right.$ until day 5 and to $29^{\circ} \mathrm{C}$ until day 10), light:dark cycle $14 \mathrm{~h}: 10 \mathrm{~h}$ and water and food ad libitum. The experiments were conducted with the same chicks at day 0 (H0; between 12 and $20 \mathrm{hs}$ ) and ten (H10) after hatching. The experimental protocols were in agreement with the guidelines of the National Council of Control in Animal Experimentation (CONCEA-MCT-Brazil) and approved by the local Animal Care and Use Committee (CEUA - \# 024166/13).

\subsection{Oxygen consumption}

$\dot{V} \mathrm{O}_{2}$ was measured using an open-flow respirometry method (Szdzuy et al., 2008). Chicks were placed individually in a respirometer (total volume: $540 \mathrm{~mL}$ for $\mathrm{H0}$; $1000 \mathrm{~mL}$ for $\mathrm{H} 10$ ) inside a temperature controlled chamber (FANEM, Sao Paulo, SP, Brazil). The ambient temperature (Ta) and gas concentrations inside the respirometer varied according to the protocol and age (see "Protocols" item for details). The incurrent air was pulled (pull mode; MFS, Sable Systems, Las Vegas, $\mathrm{NV}, \mathrm{USA}$ ) into the respirometer at a rate of $800 \mathrm{~mL} / \mathrm{min}(\mathrm{HO})$ or $1500 \mathrm{~mL} / \mathrm{min}$ (H10) and the gases concentrations were monitored intermittently. Outflow air passed through a drying column (Drierite, Sigma Aldrich, St. Louis, MO, USA), was subsampled $(180 \mathrm{~mL} / \mathrm{min}$; SS4, Sable Systems, Las Vegas, NV, USA) and finally pulled through calibrated analyzers for O2 (PA-10; Sable Systems, Las Vegas, NV, USA) and CO2 (CA-10; Sable Systems, Las Vegas, NV, USA) concentrations recording. The $\dot{V} 0_{2}\left(\mathrm{~mL} \mathrm{O} 2 / \mathrm{kg} \cdot \mathrm{min}^{-1} \mathrm{STPD}\right)$ was calculated using the following equation (Depocas and Hart, 1957; Lighton, 2008, Eq. 11.7):

$\dot{V}_{o_{2}}=\frac{F R_{\mathrm{e}}\left[\left(F_{i} \mathrm{O}_{2}-F_{e} \mathrm{O}_{2}-\right)-F_{i} \mathrm{O}_{2}\left(F_{e} \mathrm{O}_{2}-F_{i} \mathrm{O}_{2}-\right)\right]}{1-F_{i} \mathrm{O}_{2}}$

where:

FRe excurrent flow rate;

$\mathrm{FiO} 2$ incurrent fractional concentration of oxygen (from baseline);

FeO2 excurrent fractional concentration of oxygen;

FiCO2 incurrent fractional concentration of carbon dioxide (from baseline);

$\mathrm{FeCO} 2$ excurrent fractional concentration of carbon dioxide.

\subsubsection{Body temperature $(\mathrm{Tb})$ measurement}

Colonic temperature was measured by inserting a thin temperature sensor at $3 \mathrm{~cm}$ through the animal's cloaca. The sensor was connected to an analog thermometer (Yellow Spring Instrument, Yellow Spring, Ohio, USA).

\subsection{Thermal preference}

The thermal gradient chamber used in the present study is the same described in Vizin et al. (2015) but slightly modified to be cooled down to $15^{\circ} \mathrm{C}$ at one end of it and warmed up to $40{ }^{\circ} \mathrm{C}$ at the other end. For each experiment, a linear thermal gradient was considered acceptable if $R 2 \geq 0.96$. Pictures of chicks's position in the lanes were taken every minute by using a webcam positioned at the top of the apparatus. The temperatures selected by the animals were calculated based on the equation of the linear regression and the position of the chicks obtained from the pictures. The thermal gradient chamber has been built with eight thermometers even distributed just under the grid floor where the temperatures are slightly colder than the air temperature at mid chick height. Thus, a linear regression was calculated between the temperatures obtained under and above the grid for all thermometers and then used to correct the Ta recorded by the thermometers below the grid.

\subsection{Protocols}

2.4.1. Effect of hypoxic incubation on resting metabolic rate and $\mathrm{Tb}$ of normoxic chicks at days 0 (HO) and 10 (H10) after hatching

After having their $\mathrm{Tb}$ measured, animals of both ages ( $\mathrm{HO}$ and $\mathrm{H} 10$ ) and both incubation groups ( $\mathrm{Nx}$ and $\mathrm{Hx}$ ) were individually placed in the respirometer under normoxia $\left(20.95 \% \mathrm{O}\right.$; at Ta of $\sim 34^{\circ} \mathrm{C}$ or $30^{\circ} \mathrm{C}$ for $\mathrm{HO}$ and $\mathrm{H} 10$, respectively) for approximately $30 \mathrm{~min}$ for habituation and then the $\dot{V} O_{2}$ was determined. Following these resting measurements, animals were divided in two groups and were exposed to either hypoxia or cold (see below).

\subsubsection{Effect of hypoxic incubation on metabolic rate and Tb of chicks ex-} posed to an acute hypoxic event at days 0 (HO) and 10 (H10) after hatching

After the normoxic resting measurements, incurrent gas was switched to a hypoxic gas mixture of 15\% O2 (15\% O2 and N2 balance, White Martins, Osasco, SP, Brazil) and gases concentrations inside the respirometer were measured after $13 \mathrm{~min}$, for $2 \mathrm{~min}$. Lastly, incurrent gas was once again switched to 10\% 02 (10\% 02 and N2 balance, White Martins, Osasco, SP, Brazil) and gas measurements were repeated after $13 \mathrm{~min}$, for $2 \mathrm{~min}$. As mentioned, Tb was measured before they were placed into the respirometer (initial $\mathrm{Tb}$ ) and right after they were removed from there (after the $10 \%$ hypoxia exposure; final $\mathrm{Tb}$ ). 
2.4.3. Effect of hypoxic incubation on metabolic rate and Tb of chicks exposed to different Ta at days 0 (HO) and 10 (H10) after hatching

In the second group of chicks, after the normoxic resting measurements, the Ta inside the chamber was increased to $38.5{ }^{\circ} \mathrm{C}(\mathrm{H} 0)$ or $33{ }^{\circ} \mathrm{C}$ (H10) for about $20 \mathrm{~min}$ and then decreased in a stepwise way $\left(2{ }^{\circ} \mathrm{C}\right.$ each $\left.10 \mathrm{~min}\right)$. The actual Ta obtained inside the respirometer, from 38.5 to $29{ }^{\circ} \mathrm{C}$ for $\mathrm{HO}$ and from 33 to $24^{\circ} \mathrm{C}$ for $\mathrm{H} 10$ (which are the Ta presented in the Fig. 3), were slightly different from the Ta set at the temperature controlled chamber's panel. $\mathrm{O} 2$ and $\mathrm{CO} 2$ concentrations were registered for $2 \mathrm{~min}$, but the measurements only started 8 min after the animals were exposed to the new Ta because that was the time required for the respirometer to equilibrate at the Ta of interest. The total interval of the experiment was $2 \mathrm{~h}$ (the needed time to achieve the lowest Ta). The Ta inside the respirometer was measured by a datalogger (Subcue, Calgary, AB, Canada) and the Tb was measured before animals were placed into the respirometer (initial $\mathrm{Tb}$ ) and right after they were removed from there (final $\mathrm{Tb}$ ).

\subsubsection{Effect of hypoxic incubation on thermal preference of H10 chicks in} normoxia and exposed to hypoxia

Chicks were habituated to the thermal gradient chamber for $1 \mathrm{~h} /$ day for 3 days before the experiment (from the 7 th to the 9 th day). At the experimental day, $\mathrm{H} 10$ chicks were positioned at the middle of the lanes and their thermal preference was recorded for $1 \mathrm{~h}$ in normoxia followed by $30 \mathrm{~min}$ in hypoxia ( $15 \pm 0.5 \%$ of 02 ). Hypoxia was induced by pushing pure $\mathrm{N} 2$ into the thermocline from both ends. The concentration of oxygen in the middle of the thermocline was monitored by an $\mathrm{O} 2$ analyzer (Oxystar-100 02 monitor, CWE Inc. Ardmore, PA, USA). For this protocol, chicks were placed in pairs in each lane of the thermocline because, based on our previous tests, animals that have hatched together reacted by continuously calling when they were individually placed into the lanes. Being in pairs in the thermal gradient seems not to interfere with thermal preference, according to a previous study (Toro-Velasquez et al., 2014).

\subsection{Statistical analysis}

Data are presented as means \pm SEM. The characteristics of the eggs and body mass of the chicks (Table 1 ) were compared by unpaired $t$ test. The effect of incubation treatment on oxygen consumption and Tb was analyzed by repeated measures (RM) two-way ANOVA [factors: age ( $\mathrm{HO}$ and $\mathrm{H} 10)$ and incubation treatment ( $\mathrm{Nx}$ and $\mathrm{Hx}$ ); Fig. 1]. The effects of acute hypoxia and of different ambient temperatures on oxygen consumption in each age were analyzed by repeated measures (RM) two-way ANOVA (factors: hypoxia or ambient temperature and incubation treatment; Figs. 2A; B; 3A; B). Whenever RM ANOVA resulted in significant main or interaction effects, a Holm-Šidák post hoc test was performed to verify where the differences existed (Seaman et al., 1991). For data that were not normally distributed or homogeneous, log transformation was use, and in cases where log transformation was insufficient in terms of model assumptions, ranked data were analyzed. The reductions in Tb under acute hypoxia and under different ambient temperatures exposures of chicks from $\mathrm{Nx}$ and $\mathrm{Hx}$ incubations where compared by unpaired $t$-test (for parametric data) or Mann-

Table 1

Characteristics of the eggs and chicks.

\begin{tabular}{lll}
\hline & $\mathrm{Nx}$ & $\mathrm{Hx}$ \\
\hline Number of hatchlings & 18 & 17 \\
Fresh egg mass, g & $61.5 \pm 0.3$ & $61.0 \pm 0.2$ \\
Incubation age at hatching & $20.4 \pm 0.05$ & $20.3 \pm 0.06$ \\
Body mass (g), H0 & $45.2 \pm 0.4$ & $43.8 \pm 0.4^{\mathrm{a}}$ \\
Body mass (g), H10 & $155.3 \pm 5.4$ & $147.8 \pm 8.2$
\end{tabular}

$\mathrm{Nx}=$ normoxia incubation; $\mathrm{Hx}=$ hypoxia incubation. $\mathrm{H} 0=$ hatchling (between 12 and $20 \mathrm{hs}) ; \mathrm{H} 10=$ ten days old chicks. Data are means \pm SEM.

Means significant difference from $\mathrm{Nx}$

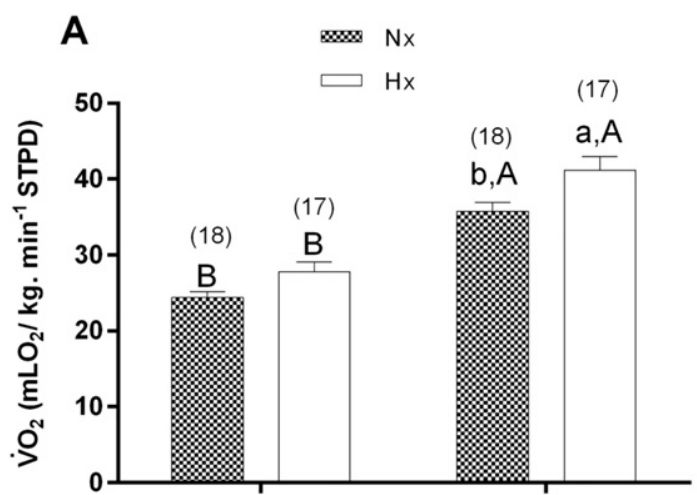

B

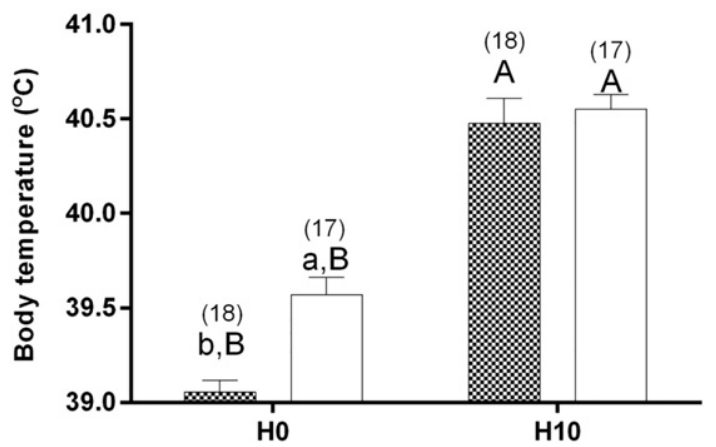

Fig. 1. Effect of hypoxia $\left(15 \% \mathrm{O}_{2}\right)$ during the last third of incubation $(\mathrm{Hx})$ on resting metabolic rate $\left(\mathrm{VO}_{2} ; \mathrm{A}\right)$ and body temperature $(\mathrm{B})$ in hatchlings $(\mathrm{HO})$ and 10 day-old chicks $(\mathrm{H} 10) . \mathrm{Nx}=$ normoxic incubation. $\mathrm{Hx}=$ hypoxic incubation. Different lowercase letters indicate significant difference between treatments within the same age; different uppercase letters represent significant difference between ages within the same treatment. Numbers in parenthesis depict experimental $n$.

Whitney Rank Test (for non-parametric data) (Figs. 2C; D; 3C; D). Chi square test was used to compare frequency distribution data obtained in the thermal gradient similarly to what was recently done by Cecchetto and Naretto (2015). Differences were considered significant when $p \leq 0.05$.

\section{Results}

Although the age at hatching was not affected, the body mass (BM) of hatchlings was reduced by hypoxic treatment during the last week of incubation ( $p=0.0017$; Table 1$)$. This difference on BM was not observed at 10 days after hatching.

3.1. Effect of hypoxic incubation on resting metabolic rate and $\mathrm{Tb}$ of normoxic chicks at days 0 (HO) and 10 (H10) after hatching

Both age and hypoxia exposure affected resting metabolic rate of chicks. Regardless the incubation treatment, the oldest animals had higher $\dot{V} 0_{2}$ than the hatchlings (effect of age: $p<0.001$. Fig. $1 \mathrm{~A}$ ). Additionally, further increase on $\dot{V} 0_{2}$ was observed in $\mathrm{H} 10$ incubated under hypoxia compared to those of same age incubated under control conditions, but no hypoxic effect was found in $\mathrm{HO}$ (effect of hypoxia: $p=$ 0.003; no interaction effect. Fig. $1 \mathrm{~A}$ ). Regarding Tb, age affected it similarly to $\dot{V} O_{2}$, the oldest animals had higher Tb compared to the youngest ones (effect of age: $p<0.001$ Fig. 1B). Nevertheless, the hypoxia effect was only detected on $\mathrm{H} 0$ : hatchlings incubated under $15 \%$ of $\mathrm{O} 2$ showed higher Tb than the control ones (effect of incubation: $p=0.005$. Fig. 1B). 
A

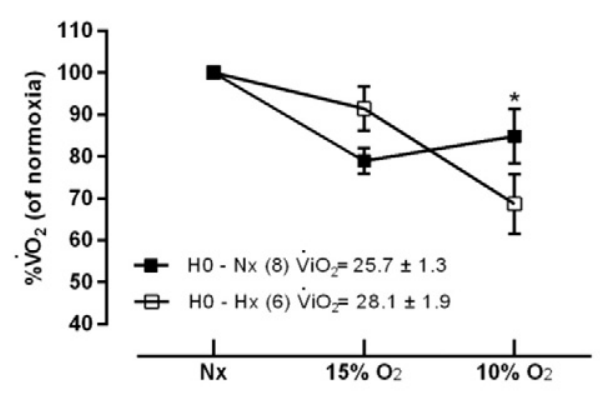

C

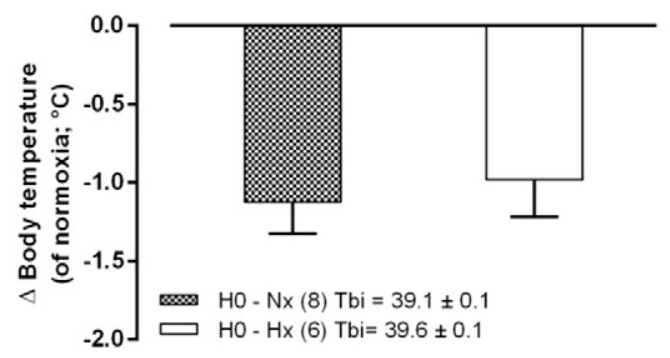

B

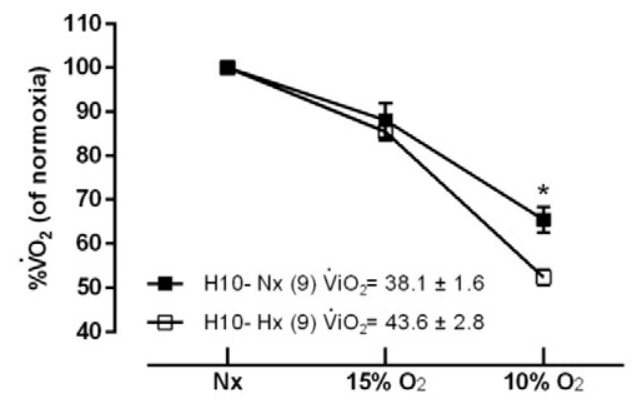

D

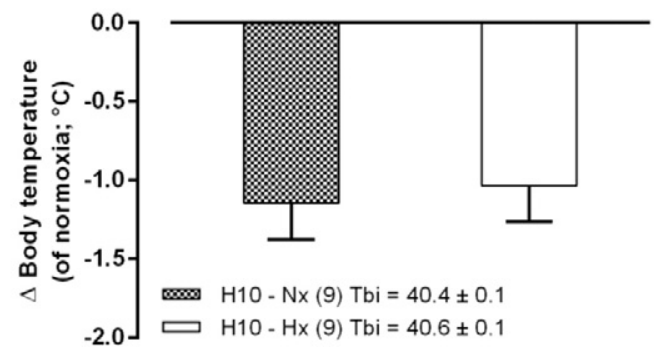

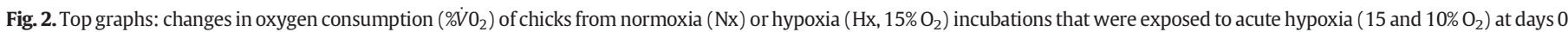

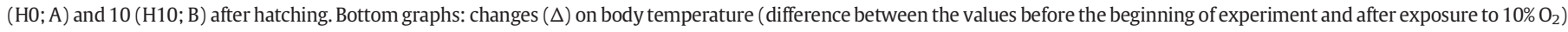

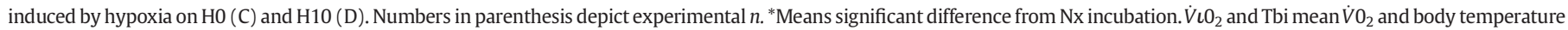
before hypoxia exposure.

A

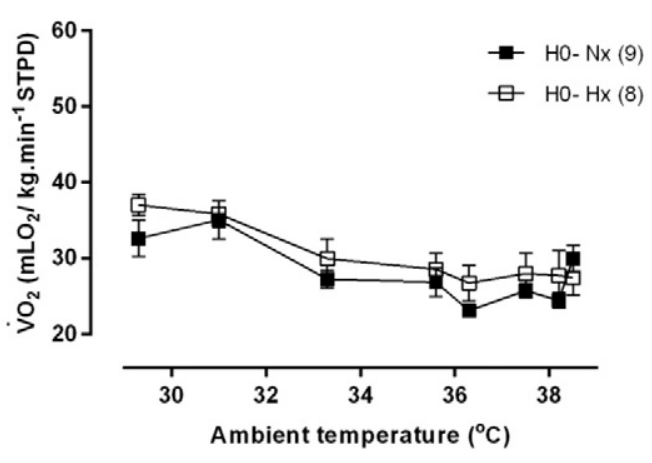

C

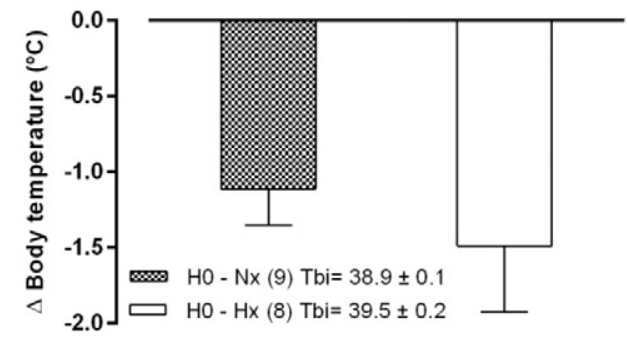

B

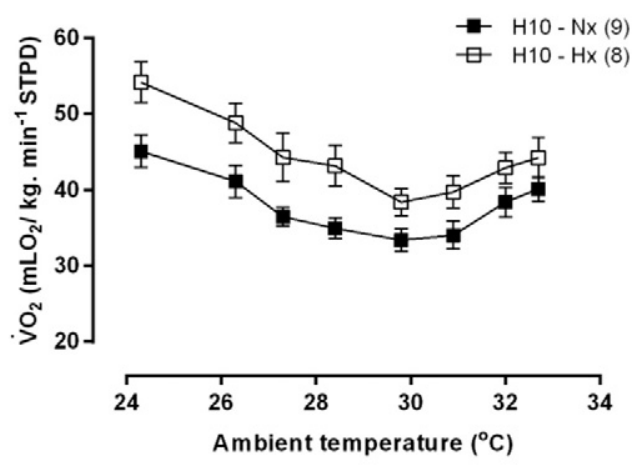

D

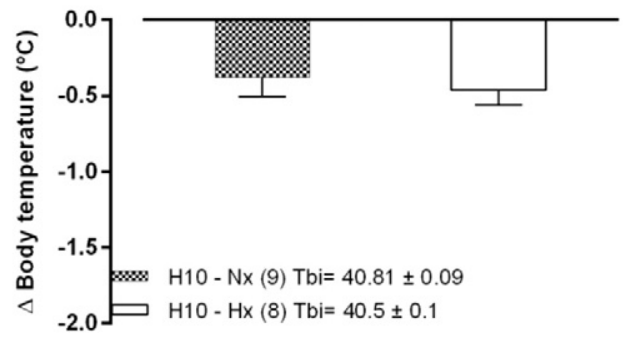

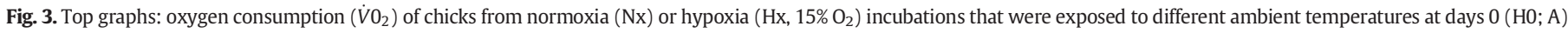

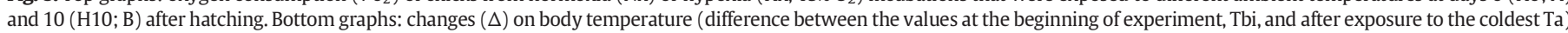
induced by cold on H0 (C) and H10 (D). Numbers in parenthesis depict experimental $n$. *Means significant difference from Nx incubation. 
3.2. Effect of hypoxic incubation on metabolic rate and Tb of chicks exposed to an acute hypoxic event at days 0 (HO) and 10 (H10) after hatching

Acute exposure of $\mathrm{H} 0$ to both moderate and severe hypoxia (15\% and $10 \%$, respectively, for 15 min each) similarly reduced $\dot{V} 0_{2}$ on those animals incubated under normoxic conditions; however only the severe level of hypoxia reduced the $\dot{\mathrm{V}} \mathrm{O}_{2}$ of those $\mathrm{H} 0$ incubated under low $\mathrm{O} 2$ atmosphere (interaction effect: $p=0.015$; Fig. 2A). For H10, both hypoxia levels gradually reduced the $\dot{V} \mathrm{O}_{2}$ of all animals ( $\mathrm{Nx}$ and $\mathrm{Hx}$ incubations), but the $10 \% \mathrm{O} 2$ exposure caused a further reduction in $\dot{V} 0_{2}$ in animals from Hx incubation (interaction effect: $p=0.004$. Fig. $2 B$ ). The $\mathrm{Tb}$ of all animals of both ages was equally reduced by hypoxia $(10 \% 02)$ regardless the treatment during the incubation (no treatment effect: $p=0.654$ for $\mathrm{H} 0$ and $p=0.739$ for $\mathrm{H} 10$; no interaction effect. Fig. $2 \mathrm{C}$ and $\mathrm{D})$.

3.3. Effect of hypoxic incubation on metabolic rate and Tb of chicks exposed to different Ta at days 0 (HO) and 10 (H10) after hatching

The decrease in Ta below $33^{\circ} \mathrm{C}$ caused an increase in $\dot{V} 0_{2}$ of hatchlings with no difference between treatments (effect of Ta: $p<0.001$; no interaction effect; Fig. 3A). When they were older (H10), chicks also presented an increase in oxygen consumption when exposed to cold and warm Tas; however those animals incubated under hypoxia presented a higher $\dot{V} O_{2}$ compared to control ones (effect of incubation: $p=0.03$; effect of Ta: $p<0.001$, no interaction effect. No difference between $\mathrm{Nx}$ and $\mathrm{Hx}$ at $\mathrm{Ta}=29.8,32,32.7^{\circ} \mathrm{C}$; Fig. 3B).

Body temperature of $\mathrm{HO}$ and $\mathrm{H} 10$ was similarly reduced after cold exposure for both incubation treatments (HO: no treatment effect: $p=1.000$; Fig. 3C. H10: no treatment effect: $p=0.614$; Fig. 3D).

\subsection{Effect of hypoxic incubation on thermal preference of H10 chicks in normoxia and exposed to hypoxia}

Fig. 4 depicts the frequency distribution of thermal preference of 10 day-old chicks incubated in normoxia and in hypoxia. The Hx chicks selected ambient temperatures close to $30.5{ }^{\circ} \mathrm{C}$ ( $92 \%$ of time), which were warmer than the thermal range selected by the Nx chick, between 24 and $27^{\circ} \mathrm{C}(\chi 2=200.000$; d.f. $=6 ; p<0.001$; Fig. 4). Both groups reduced thermal preference when exposed to $15 \% \mathrm{O} 2$ for $30 \mathrm{~min}$ (For $\mathrm{Nx}$ group: $\chi 2=153.589$; d.f. $=5 ; p<0.001$. For Hx group: $\chi 2=188.161$; d.f. $=6 ; p<0.001$ ).

\section{Discussion}

In the present study, we confirmed our hypothesis that hypoxia during the last week of incubation affects the thermoregulation of chicks up to 10 days after hatching when compared to normoxic incubated chicks.

\subsection{Energy metabolism and thermoregulation of hatchlings ( $\mathrm{HO})$ exposed to hypoxia during the last week of incubation}

The exposure of embryos to hypoxia during the third week of incubation has decreased the BM of hatchlings (Table 1). Similar results have been found in other studies with fetus at the last days of incubation (Wangensteen et al., 1974; Azzam et al., 2007; Azzam and Mortola, 2007; Giussani et al., 2007) and hatchlings (Dzialowski et al., 2002; Hassanzadeh et al., 2004) that were exposed to hypoxia during prenatal stages or incubated in high altitudes. Mortola and Cooney (2008) demonstrated that chronic hypoxia during incubation reduces growth, impairing the normal development of the embryo. Such reduction on growth has been considered as a survival strategy against low 02 partial pressure because, besides saving energy, it favors the protection of some key organs at expense of delaying other tissues growth (Azzam and Mortola, 2007). In addition, the lower growth of hypoxic embryos
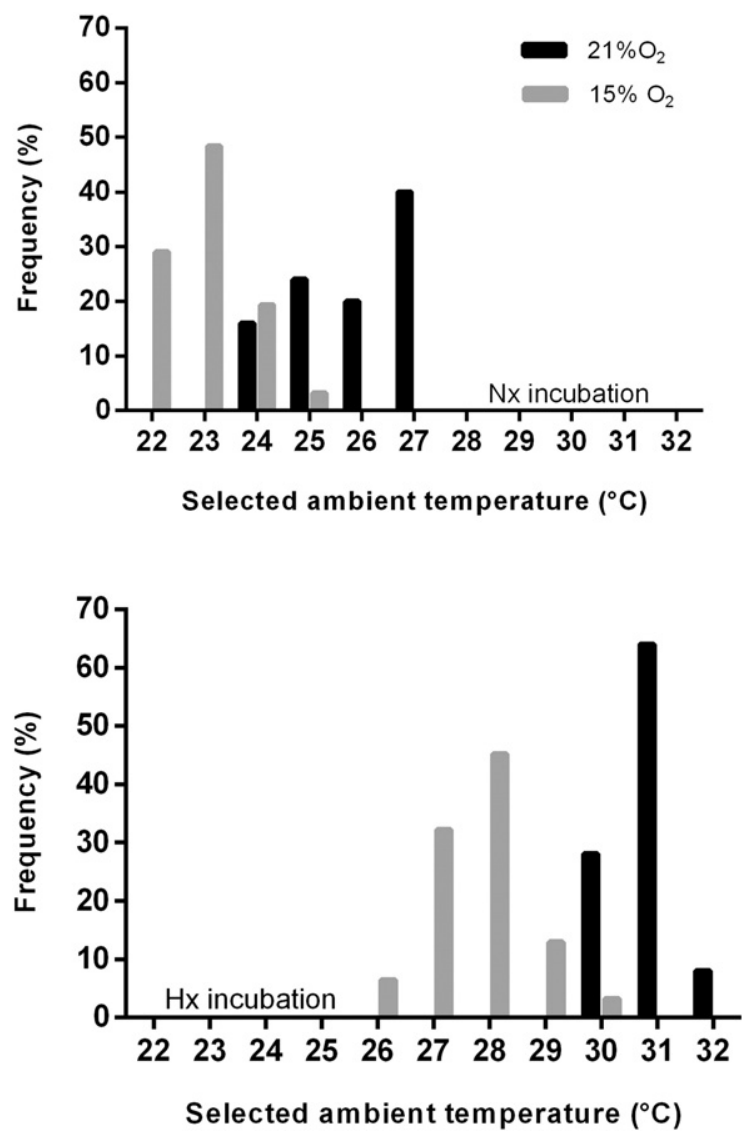

Fig. 4. Frequency of distribution of ambient temperatures selected by 10 day-old chicks in the thermal gradient during normoxia $\left(21 \% \mathrm{O}_{2}\right)$ or an acute exposure to hypoxia $\left(15 \% \mathrm{O}_{2}\right)$. Top graph: chicks from normoxic incubation. Bottom graph: chicks from hypoxic incubation $\left(\mathrm{Hx} ; 15 \% \mathrm{O}_{2}\right)$. Number of animals, $\mathrm{Nx}=6, \mathrm{Hx}=10$.

reduces the yolk consumption (Haron et al., 2016) which can explain some findings of no changes or even increases in total BM of hatchlings after hypoxic incubation (Azzam et al., 2007; Lourens et al., 2007; Ferner and Mortola, 2009; Bahadoran et al., 2010). In the present study we could not measure yolk mass separated from the body of HO chicks because we used the same animals at day 10 , but in another group of animals it was observed that the relative mass of yolk (yolk mass/body mass) was $9-11 \%$ and $15-17 \%$ for normoxia and late hypoxia incubation, respectively (personal observations), which indicates our $\mathrm{Hx}$ hatchlings from the present study were even smaller.

The absolute values of $\mathrm{Tb}$ in our $\mathrm{Nx}-\mathrm{H} 0$ chicks were lower than those of Ferner and Mortola (2009), which may be related to differences in lineages (broiler $\times$ White leghorn) and/or ambient temperature ( 33$\left.34^{\circ} \mathrm{C} \times 38^{\circ} \mathrm{C}\right)$; however, they are similar to those described by Yoneta et al. (2007; broilers and White leghorn at $35^{\circ} \mathrm{C}$ ) and Szdzuy and Mortola (2007; White leghorn at $37.5^{\circ} \mathrm{C}$ ). Actually, Tzschentke and Nichelmann (1999) shows Tb varying from about $38.3^{\circ} \mathrm{C}$ at $30{ }^{\circ} \mathrm{C}$ to about $40^{\circ} \mathrm{C}$ at $35^{\circ} \mathrm{C}$ in chicken hatchlings.

The Hx group had the same resting $\dot{V} 0_{2} / \mathrm{kg}$ but higher Tb compared to normoxia-incubated chicks, which would lead one to suggest an increase in heat conservation in these animals (Fig. 1). In addition, hypoxia-incubated hatchlings showed no alteration on $\dot{V} 0_{2}$ when exposed to $15 \%$ of $\mathrm{O}_{2}$ but their metabolic rate was further reduced in response to $10 \%$ of $\mathrm{O} 2$ compared to those animals incubated under control gas, indicating that they $(\mathrm{Hx})$ had a greater metabolic reaction to hypoxia, which seems to be beneficial for survival in those conditions (Mortola et al., 1992; Mortola, 2004; Lu et al., 2005). Despite this fact, both Nx and Hx groups had similar reductions in $\mathrm{Tb}$ at $10 \%$ O2, i.e., about $1{ }^{\circ} \mathrm{C}$ (Fig. 3C), which would also suggest a higher heat conservation on $\mathrm{Hx}$ group. 
The comparison of the calculated thermal conductance between $\mathrm{Nx}$ $\left(2.62 \pm 0.09 \mathrm{~W} / \mathrm{kg} \cdot{ }^{\circ} \mathrm{C}\right)$ and $\mathrm{Hx}\left(2.59 \pm 0.09 \mathrm{~W} / \mathrm{kg} \cdot{ }^{\circ} \mathrm{C}\right)$ groups, however, showed no statistical difference ( $t$-test; $p=0.85)$. In this case, a probable higher $\dot{V} \mathrm{O}_{2} / \mathrm{kg}$ in $\mathrm{Hx}$ compared to control hatchlings, had body weight excluded the yolk mass, could be an explanation for the higher $\mathrm{Tb}$ in $\mathrm{Hx}$ chicks.

When the Ta was reduced to $31{ }^{\circ} \mathrm{C}$ or below it, all $\mathrm{HO}$ had their $\dot{V} \mathrm{O}_{2}$ slightly increased as expected for a thermoregulatory response to cold (Mortola and Frappell, 2000; Mortola and Maskrey, 2011), with no difference between incubation groups, suggesting that low 02 partial pressure caused no damage to the animal's thermogenesis ability. Similar results have been observed in White leghorn hatchlings incubated under hypoxia and exposed to cold after hatching (Azzam et al., 2007). Also in rats, it was demonstrated that animals submitted to prenatal hypoxia do not present different metabolic responses to changes in Ta (from 15 to $40^{\circ} \mathrm{C}$ ) compared to control animals (Mortola and Naso, 1998). Regardless of the incubation treatment, the $\mathrm{Tb}$ of all our hatchlings was similarly reduced after cold exposure, as chicken at this age are known by having no homoeothermic range of Tb despite the weak cold-induced thermogenesis (Tzschentke and Nichelmann, 1999).

4.2. Energy metabolism and thermoregulation of 10-day old chicks (H10) exposed to hypoxia during the last week of incubation

The H10 chicks incubated under hypoxia had no difference on BM compared to control ones (Table 1), despite their reduced BM when they were hatchlings. This seemed to be result of a "catch-up" growth, i.e., after a transitional period of growth inhibition, animals usually present a higher growth rate than the control ones and then BM is equalized between treatments (Boersma and Wit, 1997). Similar results had also been described in infants and neonate rats induced by a variety of different growth-retarded stimuli (Prader et al., 1963; Gingell et al., 1981; Okubo and Mortola, 1988; Sant'Anna and Mortola, 2003).

All ten day-old animals (from $\mathrm{Nx}$ and $\mathrm{Hx}$ incubations) presented a higher resting $\dot{V} \mathrm{O}_{2} / \mathrm{kg}$ compared to when they were hatchlings (Fig. $1 \mathrm{~A})$. This is in agreement with the data in chickens that show an increase in mass specific metabolic rate until around ten days old followed by an allometric decrease in $\dot{V} O_{2} / \mathrm{kg}$ (Bobek et al., 1977; Snyder et al., 1991; Espinha et al., 2014). Interestingly, our Hx chicks showed a higher $\dot{V} 0_{2} / \mathrm{kg}$ at the age of 10 days compared to the Nx group (Fig. $1 \mathrm{~A}$ ), which seems to be well related with high activity involved in a catch up growth. In this context, thyroid hormones are good candidates for mediate such metabolic changes. Evidence in mammals exists showing that lambs whose mothers had placental restriction presented faster growth and higher plasma T3 levels in the first month of postnatal life (Blasio et al., 2006). Besides their role in energy metabolism, the hypothalamus-hypophysis-thyroid axis is well known to be involved in growth in chicken (Kühn et al., 2005). Higher plasma concentration of T3 hormone in Hx than Nx chicks ( $2.7 \pm 0.1$ vs $2.1 \pm 0.2 \mathrm{ng} / \mathrm{mL} ; n=$ $10 ; p=0.003$, Mann-Whitney Rank Sum Test) was indeed observed by us in another group of animals used for other purposes.

Body temperature was also higher in $\mathrm{H} 10$ than $\mathrm{H} 0$ possibly due to the increase in $\dot{V} \mathrm{O}_{2}$ and the decrease in surface/volume ratio as the animals become bigger and older (maturation of thermoregulation). Despite the fact the hypoxia-incubated $\mathrm{H} 10$ chicks had higher $\mathrm{V} \mathrm{O} 2$ than the normoxic group (same age, Fig. 1A), animals of both groups had a similar Tb (Fig. 1B), which indicates that hypoxic incubation might also have decreased heat conservation/increased heat loss, in comparison to control animals at this age. The calculated thermal conductance of $\mathrm{Hx}-\mathrm{H} 10\left(1.31 \pm 0.05 \mathrm{~W} / \mathrm{kg} \cdot{ }^{\circ} \mathrm{C}\right)$ was indeed higher than that of $\mathrm{Nx}-$ $\mathrm{H} 10\left(1.14 \pm 0.05 \mathrm{~W} / \mathrm{kg} \cdot{ }^{\circ} \mathrm{C}\right)(t$-test; $p=0.03)$. The results about cold exposure of $\mathrm{H} 10$ chicks are in agreement with this, as Hx group had higher $\dot{V} 0_{2}$ at lower temperatures than Nx chicks (Fig. 3B and D). Moreover, by calculating the linear regression connecting minimal $\dot{V} 0_{2}$ at $29.8^{\circ} \mathrm{C}$ with the $\mathrm{Ta}=\mathrm{Tb}$ value at $\mathrm{X}$ axis $\left(\dot{V} \mathrm{O}_{2}=0\right)$, it was possible to observe a higher slope of the Hx compared to Nx line ( $p=0.02$; unpaired $t$-test), indicating a higher conductance of chicks incubated in hypoxia. A similar result, i.e., a higher slope for $\mathrm{Hx}$ ( $p=0.04$; unpaired $t$-test), is observed even if the linear regression is calculated including the $\dot{V} 0_{2}$ at 26$28^{\circ} \mathrm{C}$ with the $\mathrm{Ta}=\mathrm{Tb}$ value at $\mathrm{X}$ axis.

The possibility of a reduced insulation of $\mathrm{H} 10$ chicks incubated under hypoxia is also supported by the results of behavioral thermoregulation. In spite of higher metabolic rate (Fig. 1), hypoxia-incubated $\mathrm{H} 10$ animals selected higher Tas in the thermal gradient than the control ones under 21\% O2 (Fig. 4), indicating they select a condition that diminish the differences between core and ambient temperatures, trying to reduce heat loss (Almeida et al., 2006; Vizin et al., 2015). Our results contrast with those of Azzam et al. (2007), who did not find any difference in preferred Tb of leghorn hatchlings submitted to hypoxia from the 5th day to the end of incubation. The distinct results between the two studies may be related to differences in lineage, interval of hypoxia exposure during incubation and age of chicks whose thermal preference was tested.

Interestingly, although different individuals were used for the thermocline (Fig. 4) and the $\dot{V} O_{2}$ in the cold experiments (Fig. 3), it seems that the selected Tas by normoxic Nx chicks may be below the thermoneutral zone (TNZ). The lowest metabolic rates were found at Tas between $\sim 27$ and $\sim 31.5^{\circ} \mathrm{C}$ (Fig. 3) while the selected Tas by Nx chicks was between 24 and $27^{\circ} \mathrm{C}$ (Fig.4). Such observation is similar to that found in leghorn hatchlings, which prefer Ta below their TNZ (Toro-Velasquez et al., 2014), but this subject needs further investigation to be confirmed in older chicks.

As expected, both groups selected lower Tas during acute hypoxia exposure, which is considered a response that favors Tb reduction during low oxygen condition (Gordon and Fogelson, 1991; Gordon, 1997). One may argue that the results of thermal preference were influenced by keeping two animals at the same lane of the thermal gradient, but this may not be the case because, at least in hen hatchlings, being alone or in pairs makes no difference in the thermal preference (Toro-Velasquez et al., 2014).

A possibility exist that ten day-old chicks incubated in hypoxia had increased ventilation (to match $\dot{V} O_{2}$ and avoid hypocapnia; Mortola and Maskrey, 2011), which in turn may lead to a higher evaporative heat loss contributing to keep the same Tb despite the higher $\dot{V} 0_{2}$ compared to control animals. Nevertheless, this hypothesis needs to be confirmed experimentally.

Regarding the acute hypoxia exposure, chicks from both incubation treatments showed a similar decrease in metabolic rate under $15 \%$ of oxygen, but when exposed to $10 \% \mathrm{O} 2$, those hypoxic-incubated animals showed a further metabolic drop (Fig. 2).

Under the more severe hypoxia, the metabolic depression followed a pattern observed in several species of mammals, in different ages and ambient conditions, which is the higher normoxic resting $\dot{V} 0_{2}$ the higher hypoxic metabolic drop (Bishop et al., 2001; Mortola, 2004; Mortola and Maskrey, 2011). This response seems to be well associated with the similar decrease in $\mathrm{Tb}$ of both groups at the end of the acute hypoxia exposure, although it has to be considered that 30 min (15\% followed by $10 \%$ O2) may not be enough for completing Tb decrease induced by metabolic drop. An alternative explanation could be that further oxygen consumption drop may have induced a further reduction in ventilatory response to hypoxia in Hx animals (Wood, 1991), which might have diminished the respiratory heat loss and contributed to a similar reduction on $\mathrm{Tb}$ between groups; again this is an issue that needs more studies to be clarified.

In summary, our results indicate that hypoxia during the last week of embryonic development seems to alter the growth, and then, energy metabolism and heat loss mechanisms impacting thermoregulation during the first 10 days of post-hatch life in chicken.

\section{Acknowledgement}

This study was supported by the Fundação de Amparo à Pesquisa do Estado de São Paulo-FAPESP (2010/20285-1). L.A.S. and P.A.T.V. were 
the recipient of FAPESP master (2014/01861-2) and PhD (2011/075090 ) fellowships, respectively (Animal Science Post Graduation program at FCAV-UNESP-Jaboticabal). C.S.S. was the recipient of a FAPESP PhD fellowship (2011/19131-2; Joint Graduate Program in Physiological Sciences - PIPGCF - from UFSCar/UNESP). We thank Dr. J. Mortola for insightful discussion on the manuscript.

\section{References}

Almeida, M.C., Steiner, A.A., Branco, L.G.S., Romanovsky, A.A., 2006. Cold-seeking behavior as a thermoregulatory strategy in systemic inflammation. Eur. J. Neurosci. 23, 3359-3367.

Azzam, M.A., Mortola, J.P., 2007. Organ growth in chicken embryos during hypoxia: implications on organ "sparing" and "catch-up growth". Respir. Physiol. Neurobiol. 159, $155-162$.

Azzam, M.A., Szdzuy, K., Mortola, J.P., 2007. Hypoxic incubation blunts the development of thermogenesis in chicken embryos and hatchlings. Am. J. Phys. Regul. Integr. Comp. Phys. 292, R2373-R2379.

Bahadoran, S., Hassanzadeh, M., Zamanimoghaddam, A.K., 2010. Effect of chronic hypoxia during the early stage of incubation on prenatal and postnatal parameters related to ascites syndrome in broiler chickens. Iran. J. Vet. Res. 11, 64-71.

Bicego, K.C., Barros, R.C.H., Branco, L.G.S., 2007. Physiology of temperature regulation: comparative aspects. Comp. Biochem. Physiol. A Mol. Integr. Physiol. 147, 616-639.

Bishop, B., Silva, G., Krasney, J., Nakano, H., Roberts, A., Farkas, G., Rifkin, D., Shucard, D. 2001. Ambient temperature modulates hypoxic-induced changes in rat body temperature and activity differentially. Am. J. Phys. Regul. Integr. Comp. Phys. 280, 1190-1196.

Blasio, M.J.D., Gatford, K.L., Robinson, J.S., Owens, J.A., 2006. Placental restriction alters circulating thyroid hormone in the young lamb postnatally. Am. J. Phys. Regul. Integr. Comp. Phys. 291, 1016-1024.

Bobek, S., Jastrzebski, M., Pietras, M., 1977. Age-related changes in oxygen consumption and plasma thyroid hormone concentration in the young chicken. Gen. Comp. Endocrinol. 31, 169-174.

Boersma, B., Wit, J.M., 1997. Catch-up growth. Endocr. Rev. 18, 646-661.

Cecchetto, N.R., Naretto, S., 2015. Do sex, body size and reproductive condition influence the thermal preferences of a large lizard? A study in Tupinambis merianae. J. Therm. Biol. 53, 198-204.

Chan, T., Burggren, W., 2005. Hypoxic incubation creates differential morphological effects during specific developmental critical windows in the embryo of the chicken (Gallus gallus). Respir. Physiol. Neurobiol. 145, 251-263.

Depocas, F., Hart, J.S., 1957. Use of the Pauling oxygen analyzer for measurement of oxygen consumption of animals in open-circuit systems and in a short-lag, closed-circuit apparatus. J. Appl. Physiol. 10, 388-392.

Dzialowski, E.M., Plettenberg, D.V., Elmonoufy, N.A., Burggren, W.W., 2002. Chronic hypoxia alters the physiological and morphological trajectories of developing chicken embryos. Comp. Biochem. Physiol. A 131, 713-724.

Espinha, L.P., Souza, F.A., Capalbo, A.C., Bícego, K.C., Macari, M., Gargaglioni, L.H., 2014. Age and gender influence the cardiorespiratory function and metabolic rate of broile chicks during normocapnia and hypercapnia. Respir. Physiol. Neurobiol. 200, 50-56.

Ferner, K., Mortola, J.P., 2009. Ventilatory response to hypoxia in chicken hatchlings: a developmental window of sensitivity to embryonic hypoxia. Respir. Physiol. Neurobiol. $165,49-53$.

Gautier, H., 1996. Interactions among metabolic rate, hypoxia, and control of breathing. J. Appl. Physiol. 81, 521-527.

Gingell, R.L., Pieroni, D.R., Hornung, M.G., 1981. Growth problems associated with congenital heart disease in infancy. In: Lebenthal, E. (Ed.), Gastroenterology and Nutrition in Infancy. Vol. 2. Raven Press, New York, NY, pp. 853-860.

Giussani, D.A., Salinas, C.E., Villena, M., Blanco, C.E., 2007. The role of oxygen in prenatal growth: studies in the chick embryo. J. Physiol. 585, 911-917.

Gordon, C.J., 1997. The role of behavioral thermoregulation as a thermoeffector during prolonged hypoxia in the rat. J. Therm. Biol. 22, 315-324.

Gordon, C.J., Fogelson, L., 1991. Comparative effects of hypoxia on behavioral thermoreg ulation in rats, hamsters, and mice. Am. J. Phys. 260, 120-125.

Haron, A., Dahan, Y., Shinder, D., Druyan, S., 2016. Physiological effects of hypoxic conditions during the plateau period on the chicken embryo. Comp. Biochem. Physiol. A 203, 32-39.

Hassanzadeh, M., Fard, M.H.B., Buyse, J., Bruggeman, V., Decuypere, E., 2004. Effect of chronic hypoxia during embryonic development on physiological functioning and on hatching and post-hatching parameters related to ascites syndrome in broiler chickens. Avian Pathol. 33, 558-564.
Kühn, E.R., Geelissen, S.M.E., Van der Geyten, S., Darras, V.M., 2005. The release of growth hormone $(\mathrm{GH})$ : relation to the thyrotropic- and corticotropic axis in the chicken. Domest. Anim. Endocrinol. 29, 43-51.

Lighton, J.R.B., 2008. Flow-through respirometry: the equations. In: Lighton, J.R.B. (Ed.), Measuring Metabolic Rates. A Manual for Scientists. Oxford University Press, New York, pp. 100-104.

Lourens, A., Van den Brand, H., Heetkamp, M.J.W., Meijerhof, R., Kemp, B., 2007. Effects of eggshell temperature and oxygen concentration on embryo growth and metabolism during incubation. Poult. Sci. 86, 2194-2199.

Lu, G.W., Yu, S., Li, R.W., Cui, X.Y., Gao, C.Y., 2005. Hypoxic preconditioning a novel intrinsic cytoprotective strategy. Mol. Neurobiol. 31, 255-271.

Mortola, J.P., 2004. Implications of hypoxic hypometabolism during mammalian ontogenesis. Respir. Physiol. Neurobiol. 141, 345-356.

Mortola, J.P., 2009. Review gas exchange in avian embryos and hatchlings. Comp. Biochem. Physiol. A Mol. Integr. Physiol. 153, 359-377.

Mortola, J.P., Awam, K.A., 2010. Growth of the chicken embryo: implications of egg size. Comp. Biochem. Physiol. A Mol. Integr. Physiol 156, 373-379.

Mortola, J.P., Cooney, E., 2008. Cost of growth and maintenance in chicken embryos during normoxic or hypoxic conditions. Respir. Physiol. Neurobiol. 162, 223-229.

Mortola, J.P., Feher, C., 1998. Hypoxia inhibits cold-induced huddling in rat pups. Respir. Physiol. 113, 213-222.

Mortola, J.P., Frappell, P.B., 2000. Ventilatory responses to changes in temperature in mammals and other vertebrates. Annu. Rev. Physiol. 62, 847-874.

Mortola, J.P., Maskrey, M., 2011. Metabolism, temperature and ventilation. Compr. Physiol. 1, 1679-1709.

Mortola, J.P., Naso, L., 1998. Thermogenesis in newborn rats after prenatal or postnatal hypoxia. J. Appl. Physiol. 85, 84-90.

Mortola, J.P., Toro-Velasquez, P.A., 2013. Ventilatory response to hypoxia of the 1-day old chicken hatchling after prenatal cold-induced hypometabolism. Respir. Physiol. Neurobiol. 188, 161-164.

Mortola, J.P., Gleed, R.D., Saiki, C., 1992. Adaptation and acclimatization in the hypoxic newborn mammal. In: Speck, D.F., Dekin, M.S., Revelette, W.R., Frazier, D.T. (Eds.), Respiratory Control: Central and Peripheral Mechanisms. The University Press of Kentucky, Lexington, pp. 176-180.

Okubo, S., Mortola, J.P., 1988. Long-term respiratory effects of neonatal hypoxia in the rat. J. Appl. Physiol. 64, 952-958.

Okubo, S., Mortola, J.P., 1989. Respiratory mechanics in adult rats hypoxic in the neonatal period. J. Appl. Physiol. 66, 1772-1778.

Prader, A., Tanner, J.M., von Harnack, G.A., 1963. Catch-up growth following illness or starvation. J. Pediatr. 62, 646-659.

Sant'Anna, G., Mortola, J.P., 2003. Inter-organ unevenness and catch-up growth in rats. Growth Dev. Aging 67, 27-46.

Seaman, M.A., Levin, J.R., Serlin, R.C. 1991. New developments in pairwise multiple comparisons: some powerful and practicable procedures. Psychol. Bull. 110, 577-586.

Snyder, G.K., Byers, R.L., Kayar, S.R., 1984. Effects of hypoxia on tissue capillarity in geese. Respir. Physiol. 58, 151-160.

Snyder, G.K., Coelho, J.R., Jensen, D.R., 1991. Body temperature regulation and oxygen consumption in young chicks fed thyroid hormone. Can. J. Zool. 69, 1842-1847.

Szdzuy, K., Mortola, J.P., 2007. Ventilatory chemosensitivity of the 1-day-old chicken hatchling after embryonic hypoxia. Am. J. Phys. Regul. Integr. Comp. Phys. 293, R1640-R1649.

Szdzuy, K., Fong, L.M., Mortola, J.P., 2008. Oxygenation and establishment of thermogenesis in the avian embryo. Life Sci. 82, 50-58

Tattersall, G.J., Milsom, W.K., 2003. Transient peripheral warming accompanies the hypoxic metabolic response in the golden-mantled ground squirrel. J. Exp. Biol. 206, 33-42.

Toro-Velasquez, P.A., Bícego, K.C., Mortola, J.P., 2014. Chicken hatchlings prefer ambient temperatures lower than their thermoneutral zone. Comp. Biochem. Physiol. A Mol. Integr. Physiol. 176, 13-19.

Tzschentke, B., Nichelmann, M., 1999. Development of avian thermoregulatory system during the early postnatal period: development of the thermoregulatory set-points. Ornis Fenn. 76, 189-198.

Vizin, R.C., Scarpellini, C. da S., Ishikawa, D.T., Correa, G.M., de Souza, C.O., Gargaglioni, L.H., Carrettiero, D.C., Bícego, K.C., Almeida, M.C., 2015. TRPV4 activates autonomic and behavioural warmth defence responses in Wistar rats. Acta Physiol. 214, 275-289.

Wangensteen, O.D., Rahn, H., Burton, R.R., Smith, A.H., 1974. Respiratory gas exchange of high altitude adapted chick embryos. Respir. Physiol. 21, 61-70.

Wood, S.C., 1991. Interactions between hypoxia and hypothermia. Annu. Rev. Physiol. 53, 71-85.

Yoneta, H., Dzialowski, E.M., Burggren, W.W., Tazawa, H., 2007. Endothermic heart rate response in broiler and White Leghorn chicks (Gallus gallus domesticus) during the first two days of post-hatch life. Comp. Biochem. Physiol. A 147, 529-535. 